Caribe. A pesar de que en América Central esta epidemia no ha sido suficientemente estudiada, su efecto ha sido muy negativo y se espera que empeore. Cuatro de los seis países latinoamericanos de mayor prevalencia de infección por el VIH son centroamericanos: Belice $(2,0 \%)$, Honduras $(1,6 \%)$, Panamá $(1,5 \%)$ y Guatemala $(1,0 \%)$.

Nicaragua es el segundo país más pobre de América Latina y uno de los de mayor crecimiento demográfico de las Américas. Aunque la prevalencia estimada de la infección por el VIH en adultos era de $0,2 \%$ en 2001 , existen todas las condiciones para que la epidemia se recrudezca, entre ellas una elevada movilidad de la población y una cultura que estigmatiza el uso del condón.

En este artículo se describe la epidemia de $\mathrm{VIH} /$ sida en Nicaragua y se discuten las razones del incremento en el número de casos notificados en comparación con otros países de América Central. Para ello se tomaron en cuenta todos los casos detectados en el país entre 1987 (cuando se detectó el primero) y diciembre de 2004, según los datos del Programa Nacional de Enfermedades de Transmisión Sexual, VIH y sida.

Se observó un aumento constante en la incidencia de la infección por el VIH con una disminución entre 1993 y 1998. A partir de ese año, la incidencia aumentó abruptamente hasta llegar a ser 22 veces mayor que la observada entre 1987 y 1992. A pesar de las limitaciones del sistema de vigilancia, el número de casos ha aumentado, especialmente en las zonas urbanas y las regiones centroocidental y oriental del país, con un máximo de 54,2 por 100000 habitantes. Las provincias de Chinandega (350 000 habitantes), situada en la parte occidental de Nicaragua, y Managua (1 094000 habitantes) son las más afectadas.

La mayor parte de los casos (91\%) adquirieron la infección por la vía sexual (65\% eran heterosexuales y $26 \%$, homosexuales), mientras $4 \%$ se administraba drogas intravenosas y $3 \%$ se infectó perinatalmente.

La mayor tasa de infección se encontró en personas de 20-49 años de edad, especialmente en hombres de 20-44 años, con un pico entre 35 y 39 (incidencia de 125,6 por 100000 personas), y en mujeres de 20-34 años con un pico entre 20 y 24 (incidencia de 46,6 por 100000 personas). La relación de la infección entre hombres y mujeres fue de 3:1.

La distribución de casos de infección por el $\mathrm{VIH} /$ sida fue mayor en trabajadores de servicio, obreros, amas de casa y personas vinculadas con el comercio sexual $(32,8 \%, 17,4 \%, 16,4 \%$ y $6,0 \%$, respectivamente).

La mortalidad fue estable hasta 1999, pero aumentó desde entonces hasta 2004, cuando se introdujo en Nicaragua el tratamiento antirretroviral de gran actividad (HAART). Sin embargo, se observó cierto aumento en 2005.

La renuencia al uso del condón constituye un obstáculo para cualquier posible estrategia de prevención de la infección por el VIH.

La situación actual constituye un reto para las instituciones y la sociedad nicaragüenses. Se requiere cambiar las actitudes y prácticas de las poblaciones en riesgo para reducir la vulnerabilidad del país a la epidemia de VIH/sida. Es necesario mejorar el sistema nacional de vigilancia epidemiológica y realizar estudios de prevalencia que permitan disponer de estimados más reales del alcance de esta epidemia en el país. Para evitar la diseminación de esta infección es necesario realizar campañas educativas, especialmente en hombres y mujeres laboralmente activos. (Matute AJ, Delgado E, Amador JJ, Hoepelman AIM. The epidemiology of clinically apparent HIV infection in Nicaragua. Eur J Clin Microbiol Infect Dis. 2008;27:105-8.)

\section{La toxicidad por radioterapia y la calidad de vida en mujeres con cáncer ginecológico}

El cáncer ginecológico es una de las neoplasias malignas más frecuentes y una de sus variantes, el cáncer cervicouterino, es el segundo más frecuente en mujeres en todo el mundo, especialmente en países en desarrollo. Una vez avanzado el cáncer, su tratamiento requiere por lo general de la combinación de varios tipos de intervenciones, como la cirugía, la radioterapia y la quimioterapia. Todos estos procedimientos pueden tener efectos colaterales (estenosis vaginal, alteraciones en los intestinos, la vejiga y los ovarios, trastornos del sueño y síntomas de menopausia, entre otros) que pueden contribuir a que las pacientes tengan una peor percepción de su calidad de vida.

Se realizó un estudio prospectivo longitudinal en una cohorte de 107 mujeres de 18 a 75 años de edad con diagnóstico de cáncer cervicouterino o de endometrio para investigar la incidencia de la toxicidad aguda producida por la radioterapia en la calidad de vida percibida por las pacientes e identificar los factores predictivos asociados.

La toxicidad aguda se evaluó según los Criterios Generales de Toxicidad y las recomendaciones del Grupo de Radioterapia Oncológica, ambos del Instituto Nacional del Cáncer de los Estados Unidos de América. Para evaluar la calidad de vida se aplicó la encuesta abreviada de la Organización Mundial de la Salud antes y al terminar la radioterapia, así como durante el seguimiento clínico.

Según los resultados del análisis de regresión linear multifactorial para la salud general, hubo un efecto negativo significativo de la toxicidad de las 
radiaciones en el tracto gastrointestinal superior $(P=0,043)$ y los antecedentes de cirugía $(P=0,027)$, mientras que la disminución en el sangrado vaginal influyó positivamente en la calidad de vida percibida $(P=0,047)$. No se identificaron factores de predicción de una buena calidad de vida según los dominios físicos, psicológicos, ambientales o de las relaciones sociales.

La elevada incidencia de la toxicidad inducida por las radiaciones observada en la presente cohorte $(93,5 \%)$ se manifestó principalmente en afectaciones del tubo gastrointestinal inferior $(79,6 \%)$, seguidas de trastornos en el aparato genitourinario $(74,5 \%)$ (disuria, polaquiuria y micción imperiosa) y en el tubo gastrointestinal superior $(70,4 \%)$

Sin embargo, a pesar de la elevada incidencia de las afectaciones por la toxicidad aguda, al terminar la radioterapia las pacientes manifestaron una mayor satisfacción con su calidad de vida. Las puntuaciones más bajas se encontraron en las preguntas relacionadas con la salud en general y en el dominio ambiental.

La percepción de las pacientes acerca de su calidad de vida mejoró con el tiempo, con modificaciones significativas en las puntuaciones asignadas a los dominios físico y fisiológico, a las preguntas relacionadas con la salud general y a la calidad de vida en su conjunto. Estos cambios se asociaron con el alivio de los síntomas. En particular, el alivio del dolor se asoció con un incremento en la puntuación de la calidad de vida en los dominios físico, ambiental y de relaciones sociales, mientras que la disminución del sangrado vaginal se asoció con una mejor puntuación de la calidad de vida. Estos resultados demuestran la interrelación que existe entre los diferentes factores que pueden influir en la percepción de la calidad de vida y de la salud en general.

Durante el seguimiento se observó una mejor calidad de vida percibida en los dominios físicos y psicológicos, así como en la salud general y la puntuación dada a la calidad de vida en su conjunto. Cuántos más síntomas presentan las pacientes con cáncer ginecológico, mayores beneficios asociarán con el tratamiento y mayor será la mejoría percibida en su calidad de vida, a pesar de la toxicidad aguda que experimentarán por las radiaciones. Por consiguiente, en futuros estudios longitudinales se deben tomar en cuenta las condiciones de salud y la intensidad de los síntomas antes de la radioterapia. Vaz AF, Mendes Pinto-Neto A, Marques Conde D, Costa-Paiva L, Siani Morais S, Barros Esteves S. Quality of life and acute toxicity of radiotherapy in women with gynecologic cancer: a prospective longitudinal study. Arch Gynecol Obstet. 2008;1.)

\section{Dengue y dengue hemorrágico en las Américas: guías para su prevención y control}

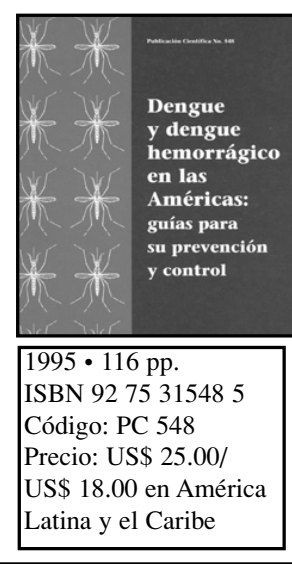

Las pautas presentadas en esta publicación subrayan el hecho de que el dengue es un problema vinculado principalmente con el saneamiento del medio domiciliario. Los vectores más importantes del dengue, Aedes aegypti y Aedes albopictus, se pueden controlar mediante maniobras físicas y sin el uso excesivo de sustancias químicas. A diferencia de los antiguos programas centralizados y de estructura vertical, estas pautas hacen hincapié en las distintas formas de transferir a la comunidad la responsabilidad, capacidad y motivación que requieren el control y la prevención del dengue.

La presente publicación, que ha sido preparada por 18 representantes de siete países de las Américas y varios funcionarios de la OPS, todos expertos en el tema tratado, ocupa un lugar crítico en la lucha contra el dengue y constituye un instrumento indispensable para profesionales de la salud que participan en el control de vectores. 